\title{
The COP9 signalosome regulates the Neurospora circadian clock by controlling the stability of the $\mathrm{SCF}^{\mathrm{FWD}-1}$ complex
}

\author{
Qun He, Ping Cheng, Qiyang He, and Yi Liu' ${ }^{1}$ \\ Department of Physiology, The University of Texas Southwestern Medical Center, Dallas, Texas 75390, USA
}

The COP9 signalosome (CSN) promotes the function of SCF-type cullin-based ubiquitin ligase complexes in vivo. Paradoxically, removal of the Nedd8 modification of cullins by CSN inhibits the ubiquitin ligase activity of SCF complexes in vitro. Ubiquitination-mediated degradation of the Neurospora circadian clock protein FREQUENCY (FRQ) is critical for clock function. Ubiquitination of FRQ requires FWD-1, the substrate-recruiting subunit of an SCF complex. Here we show that disruption of a subunit of CSN (csn-2) impairs the degradation of FRQ and compromises its normal circadian expression. A FRQ-independent oscillator drives conidiation in the csn-2 mutant, resulting in a 2-d conidiation rhythm that persists in constant darkness (DD), constant light (LL), light-to-dark (LD) transitions, and temperature cycles. Strikingly, the levels of FWD-1 are drastically reduced in csn-2 mutant, explaining the impaired degradation of FRQ.

Reduction of FWD-1 levels in the mutant requires its F-box, suggesting that its degradation is due to autoubiquitination. In addition, SKP-1 and CUL-1 of the $\mathrm{SCF}^{\mathrm{FWD}-1}$ complex are also unstable in the mutant. Therefore, our results establish an important role of CSN in the circadian clock of Neurospora. Our findings also reconcile the CSN paradox and suggest that a major function of CSN is to maintain the stability of SCF ubiquitin ligases in vivo.

[Keywords: Frequency; CSN; Smith-Magenis syndrome; FLO; proteasome; cullin; neddylation]

Received April 6, 2005; revised version accepted May 17, 2005.

Regulation of protein degradation is a critical mechanism controlling eukaryotic circadian clocks from fungi to animals (Dunlap 1999; Young and Kay 2001; Liu 2005). The circadian oscillator of the filamentous fungi Neurospora crassa, like those of the Drosophila and mammals, consists of autoregulatory negative feedback loops. In constant darkness, WHITE COLLAR-1 (WC-1) and WC-2, the two PAS domain-containing transcription factors, form a heterodimeric complex through their PAS domains and bind to the frequency (frq) promoter to activate its transcription (Crosthwaite et al. 1997; Talora et al. 1999; Cheng et al. 2001b, 2002, 2003; Froehlich et al. 2003). On the other hand, FRQ in complex with FRH, an RNA helicase, represses $f_{r} q$ transcription through its physical interactions with the WC-1/WC-2 complex (Aronson et al. 1994a; Cheng et al. 2001a, 2005; Denault et al. 2001; Merrow et al. 2001; Froehlich et al. 2003). Thus, FRQ acts as a negative element in this circadian feedback loop.

${ }^{1}$ Corresponding author.

E-MAIL Yi.Liu@UTsouthwestern.edu; FAX (214) 645-6049.

Article published online ahead of print. Article and publication date are at http://www.genesdev.org/cgi/doi/10.1101/gad.1322205.
Similar to the PERIOD (PER) proteins in Drosophila and mammals, phosphorylation-dependent degradation of FRQ is critical for clock functions (Edery et al. 1994; Garceau et al. 1997; Liu et al. 2000; Lee et al. 2001; Young and Kay 2001). Both PER and FRQ are phosphorylated and dephosphorylated by homologous kinases and phosphatases, and the phosphorylated proteins are ubiquitinated by an SCF (SKP1, Cullin, F-box)-type ubiquitin ligase and degraded by the proteasome (Lowrey et al. 2000; Lee et al. 2001; Young and Kay 2001; Grima et al. 2002; Ko et al. 2002; Lin et al. 2002; Y. Yang et al. 2002; Akten et al. 2003; He et al. 2003; Yang et al. 2003, 2004; Sathyanarayanan et al. 2004; Eide et al. 2005). Disruption of the phosphorylation or the degradation process impairs clock functions. Thus, the post-translational regulations and the degradation mechanism of clock proteins are conserved from Neurospora to animals.

Phosphorylation of FRQ is mediated by casein kinase I (CKI), CKII, and a calcium/calmodulin-dependent kinase (Gorl et al. 2001; Yang et al. 2001, 2003; Y. Yang et al. 2002). Protein phosphatase 1 (PP1) and PP2A have been shown to dephosphorylate FRQ and counteract the effects of these kinases (Yang et al. 2004). In addition to regulating the transcriptional repressor activity of FRQ, 
phosphorylation promotes FRQ degradation (Liu et al. 2000; Gorl et al. 2001; Y. Yang et al. 2002; Yang et al. 2003, 2004). Hyperphosphorylated FRQ is recognized by FWD-1, an F-box/WD40-repeat-containing adaptor protein of an SCF-type ubiquitin ligase, leading to ubiquitination and subsequent degradation (He et al. 2003). In the fwd-1 mutant strain, FRQ accumulates at high levels in its hyperphosphorylated forms, and the circadian rhythms of conidiation and gene expression in constant darkness (DD) are abolished. However, the conidiation process of the fwd-1 mutant can still be driven by light/ dark cycles. Slimb and $\beta$-TRCP, the FWD-1 homologs in Drosophila and mammals, respectively, are similarly critical for PER degradation and clock functions (Grima et al. 2002; Ko et al. 2002; Eide et al. 2005). In addition to the F-box protein (FBP), a typical SCF complex consists of a Skp1, a Cullin, and a RING domain-containing protein. Skp1-like protein bridges the interaction between FBP (through its F-box) and cullin, while the latter binds to a RING domain-containing protein that recruits the ubiquitin-conjugating enzyme.

The COP9 signalosome (CSN) is a multisubunit complex conserved in eukaryotes (Cope and Deshaies 2003; Wei and Deng 2003; Wolf et al. 2003). Since its initial discovery in Arabidopsis as an important regulator of photomorphogenesis (Wei et al. 1994), it has been implicated in a wide variety of processes in eukaryotes. CSN contains eight distinct subunits (from CSN1 to CSN8) and is structurally related to the $19 \mathrm{~S}$ lid of the 26S proteasome. Disruption of any CSN subunit is lethal in plants and animals, but not in fungi (yeast and Aspergillus) (Mundt et al. 1999, 2002; Lyapina et al. 2001; Busch et al. 2003). Interestingly, light-dependent reproduction is impaired in Aspergillus csn mutants, suggesting a conserved role of CSN in regulating light-dependent processes from fungi to plants (Busch et al. 2003).

Many of the known CSN functions are linked to its role in the ubiquitin-proteasome pathway (Schwechheimer et al. 2001). CSN is a deneddylation enzyme that cleaves the ubiquitin-like molecule, NEDD8, from the cullin subunit of SCF-type ubiquitin ligases (Lyapina et al. 2001; Cope et al. 2002). In csn mutants, cullin becomes hyperneddylated (Mundt et al. 2002; Liu et al. 2003). Deneddylation of cullin by CSN inhibits the activity of SCF ubiquitin ligase activity in vitro. Thus, CSN is a negative regulator of SCF in vitro. However, overwhelming genetic evidence indicates that CSN promotes SCF-mediated degradation of substrates in vivo (Schwechheimer et al. 2001; Cope and Deshaies 2003; Wolf et al. 2003). This apparent contradiction of CSN function in vitro and in vivo gave rise to a paradox. To reconcile this paradox, CSN had been proposed to mediate the assembly/disassembly and maintenance of cullin-containing ubiquitin ligases (Cope and Deshaies 2003; Wei and Deng 2003; Wolf et al. 2003). But the exact function of CSN in vivo remains elusive. Recently, CAND1 has been shown to specifically bind to unneddylated cullin and dissociate Skp1-FBP from the complex. Disruption of CAND1 causes phenotypes similar to those of csn mutants (Liu et al. 2002; Zheng et al.
2002; Feng et al. 2004; Goldenberg et al. 2004). In addition, UBP12, a deubiquitination enzyme, has been shown to associate with CSN and is believed to regulate the stability of F-box containing adapter proteins in fission yeast (Zhou et al. 2003).

Because of the roles of CSN in mediating light-regulated processes and in regulating SCF ubiquitin ligases, we examined its function in the Neurospora circadian clock. Here we show that FRQ degradation and clock function are impaired in a csn-2 mutant strain, suggesting that CSN is an important clock component. In addition, our data uncovered an abnormal conidiation rhythm in the csn-2 mutant. Such defects are due to low levels of FWD-1 and other SCF ${ }^{\mathrm{FWD}-1}$ components, which are caused by their rapid degradation due to autoubiquitination. Therefore, CSN is required for the stability of SCF complexes in Neurospora, reconciling the CSN paradox.

\section{Results}

Disruption of csn-2 causes growth and developmental phenotypes and hyperneddylation of CUL-1

We disrupted the Neurospora csn-2 gene (NCU00593.1) by inserting a hygromycin resistance gene $(h p h)$ into the middle of its open reading frame (ORF) using gene replacement, resulting in the disruption of its functionally important PCI (proteasome, COP9 signalsome, initiation factor 3) domain (X. Yang et al. 2002) and its C-terminal region. CSN-2 is one of the most conserved CSN subunits, and its disruption completely abolishes all known CSN function in all organisms examined (Mundt et al. 2002; Wei and Deng 2003; Schwechheimer 2004). Interestingly, the Neurospora CSN-2 is more similar to CSN2 in Arabidopsis (48\% identity) and human (49\% identity) than to CSN2 in fission yeast ( $42 \%$ identity).

Disruption of csn-2 is not lethal in Neurospora, but the homokaryotic csn-2 disruption strains (csn-2 ${ }^{\mathrm{KO}}$ ) grew more slowly and produced fewer aerial hyphae and conidia than the wild-type strain (Fig. 1A), indicating that CSN-2 is important for growth and development in Neurospora. Southern blot analysis verified the disruption of the endogenous $\operatorname{csn}-2$ gene in the $\operatorname{csn}-2^{K O}$ strains (Fig. 1B).

To confirm that CSN-2 is required for CSN function in Neurospora, we next examined the neddylation state of the Neurospora CULLIN-1 (CUL-1, NCU05204.1, 45\% identical to human CUL-1 and 30\% identical to Saccharomyces cerevisiae Cdc53p) by introducing a construct expressing c-Myc-tagged CUL-1 (Myc-CUL-1). Cullins are modified by Nedd 8 at a conserved lysine residue, causing a gel mobility retardation of cullins by $\sim 10 \mathrm{kDa}$. Because deneddylation of cullins is the only known biochemical activity of CSN (Lyapina et al. 2001; Cope et al. 2002), CUL-1 should become hyperneddylated in Neurospora mutants lacking a functional CSN. As shown in Figure 1C, two Myc-CUL-1 bands were observed in the wild-type strain. The minor band $\mid<10 \%$ of total of MycCUL-1) migrates $\sim 10 \mathrm{kDa}$ slower than the major band. 
He et al.

A

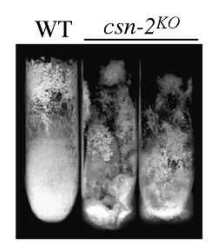

B

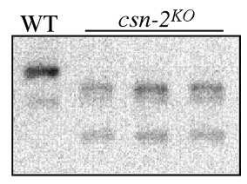

C

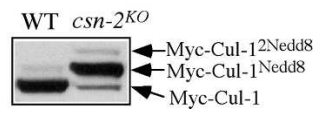

Figure 1. Disruption of $c s n-2$ leads to growth and developmental phenotypes and hyperneddylation of Cul-1. (A) The wildtype and $\operatorname{csn}-2$ mutant strains grown in minimal slants. Note that the csn-2 strains produced significantly less aerial hyphae and conidia than the wild-type strain. $(B)$ Southern blot analysis showing the disruption of the endogenous csn-2 gene in the csn-2 mutants. The genomic DNA was digested by EcoRI, and a DNA fragment containing the CSN-2 ORF was used as the probe. $(C)$ The expression profile of Myc-CUL-1 in the wild-type and $\operatorname{csn}-2^{K O}$ strains. Western blot analysis was performed using the c-Myc antibody.

The ratio of the two bands is similar to the ratios of the neddylated and deneddylated cullins in yeast (Lyapina et al. 2001; Mundt et al. 2002). Thus, the slower mobility band is likely the neddylated form of Myc-CUL-1. In contrast, most of Myc-CUL-1 is in the slower mobility forms in the csn-2 mutant strain, further suggesting that they are the products of neddylation. Interestingly, another band with molecular mass $\sim 10 \mathrm{kDa}$ greater than the neddylated Myc-Cul-1 was also observed in the mutant. This likely belongs to Myc-CUL-1 modified by two Nedd8 molecules. The dramatic difference of the MycCUL-1 neddylation pattern in these two strains indicates that CSN is not functional in the csn-2 mutant.

\section{Degradation of FRQ is impaired in the csn-2 mutant}

Because of the roles of CSN in regulating SCF-mediated protein degradation, we examined FRQ expression levels in our csn-2 $2^{K O}$ strains. As shown in Figure 2A, FRQ levels in the $\operatorname{csn}-2^{K O}$ strains were higher than in the wildtype strain (cultures in constant light, LL), and FRQ was mostly in its hyperphosphorylated forms. To examine whether the elevated FRQ levels in the mutant were due to a decrease in FRQ degradation rate, we compared the FRQ stability between the wild-type and the mutant strains following a light-to-dark (LD) transition or after the addition of the protein synthesis inhibitor, cycloheximide $(\mathrm{CHX})$ in LL. Both methods have been shown to be reliable for comparing FRQ stability (Liu et al. 1997, 2000; Gorl et al. 2001; Y. Yang et al. 2002). As shown in Figure 2B, FRQ levels in both strains decreased after a LD transition, but the rate of FRQ degradation was slower in the csn-2 mutant, suggesting that the FRQ degradation pathway is partially defective in the mutant. Surprisingly, FRQ degradation in the $c s n-2^{K O}$ strain was almost completely blocked by CHX (10 $\mu \mathrm{g} / \mathrm{mL})$ (Fig. 2C), suggesting that the residual FRQ degradation in the mutant requires protein synthesis. These results are reminiscent of what we observed in the fwd-1 $1^{R I P}$ mutant $(\mathrm{He}$ et al. 2003) except that the blockage of FRQ degradation is more severe in the $f_{w d-1}{ }^{R I P}$ mutant. Together, these data indicate that FRQ degradation is impaired in the csn-2 mutant, and the CSN-independent degradation of FRQ depends on protein synthesis.

Loss of the circadian rhythm of FRQ expression under nutrient-limiting conditions in the csn-2 mutant

The impaired FRQ degradation in the $c s n-2^{K O}$ strain prompted us to examine the circadian expression of FRQ in DD. We first performed the experiments using our standard liquid culture protocol for circadian experiments (Aronson et al. 1994a; Crosthwaite et al. 1995). The mycelia mats were first grown in LL and cut into small discs. The discs were inoculated into individual flasks containing fresh medium and transferred into DD at various time points. The cultures were then harvested at various times in DD (up to $48 \mathrm{~h}$ ). Using this protocol, all the cultures were grown in the medium for $\sim 48-60 \mathrm{~h}$ before harvest. Under these conditions, robust circadian rhythms of FRQ levels and its phosphorylation states were observed in the wild-type strain in DD (Garceau et al. 1997) (Fig. 3A). Although the FRQ rhythms in the csn-2 mutant were clearly different from those of the wild-type strain, the results were variable from experiment to experiment. In some experiments, FRQ oscilla-
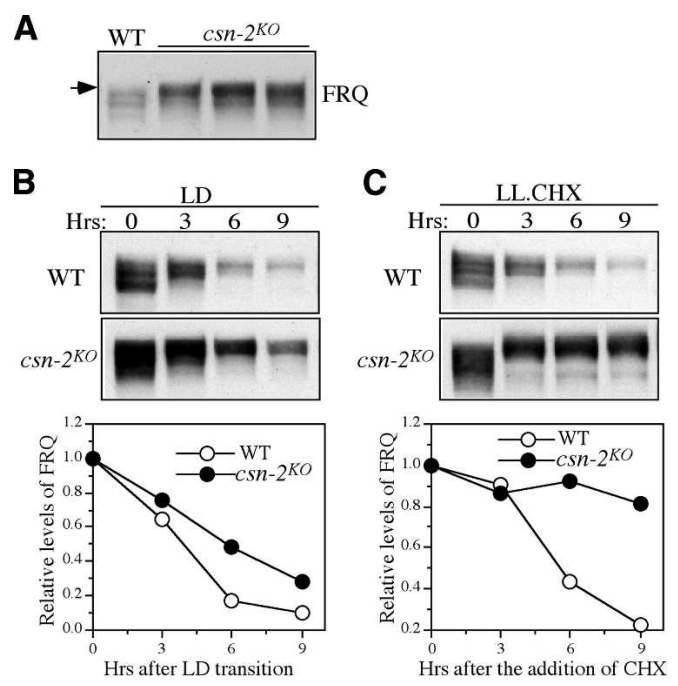

Figure 2. Degradation of FRQ is impaired in the csn-2 mutant. (A) Western blot analysis showing the expression of FRQ in the wild-type and $c s n-2^{K O}$ strains. Cultures were grown in LL. Note that FRQ levels are high and hyperphosphorylated. The arrow indicates the hyperphosphorylated FRQ species. $(B, C)$ Degradation of FRQ after a LD transition $(B)$ or the addition of CHX (10 $\mu \mathrm{g} / \mathrm{mg}$ ). (C) The bottom panels show the densitometric results of the Western blot data. Four independent experiments were performed and similar data were obtained. 
A

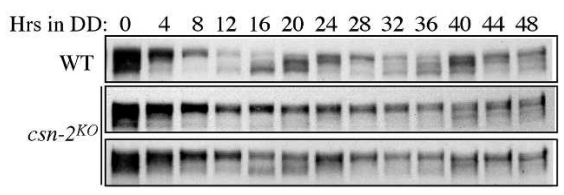

B

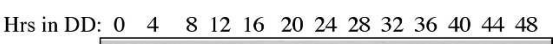

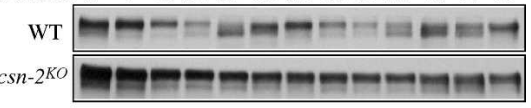

C

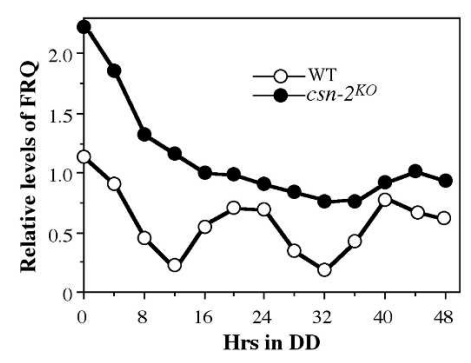

Figure 3. Loss of normal circadian expression of FRQ under normal growth $(A)$ and nutrient depletion conditions $(B, C)$ in the csn-2 mutant. Cultures were harvested at the indicated hours in DD for $2 \mathrm{~d}$. For experiments described in $A$, the cultures were grown in liquid media for $48-60 \mathrm{~h}$ before harvest, whereas in $B$ they were grown $72-84 \mathrm{~h}$ to achieve nutrient depletion. The densitometric results of the Western blot data in $B$ are shown in $C$. For experiments described in $A$, four independent experiments were performed and the results of two representative experiments are shown. For $B$, the experiments were repeated twice and similar results were obtained in both experiments.

tions appeared to be abolished in the mutant (middle panel in Fig. 3A), although a residual FRQ fluctuation was suggested by the newly synthesized hypophosphorylated FRQ species (hour 40). In other experiments, lowamplitude FRQ oscillations in its levels and phosphorylation states were evident (low panel, Fig. 3A). In all experiments, significant amounts of hyperphosphorylated FRQ were present at all times in DD in the mutant, whereas its amount oscillated robustly in the wild-type strain. Therefore, though circadian expression of FRQ is severely compromised in the csn-2 mutant for the first 2 $\mathrm{d}$ in $\mathrm{DD}$, residual FRQ oscillations can still persist with low amplitude.

Because the CSN-insensitive FRQ degradation in the csn-2 mutant requires active protein synthesis, we reasoned that the residual and inconsistent FRQ oscillations in this mutant were due to this partial functional FRQ degradation pathway. If so, FRQ oscillations should be completely abolished when protein synthesis is slowed down. To test this hypothesis, we examined FRQ oscillations under a nutrient-depleting condition that results in the decrease of cell growth and overall protein synthesis. We used a similar protocol as above except that the mycelia discs were grown in LL for additional 24 $\mathrm{h}$ before their transfer into DD. Thus, the cultures were grown in the fresh media for $72-84 \mathrm{~h}$ before harvest. The extended culturing time resulted in greater cell mass, the depletion of nutrients from the medium, and slower growth rate. As shown in Figure 3B, FRQ rhythms were not affected in the wild-type strain under this condition (the less-resolved FRQ forms were due to shorter electrophoresis time). In contrast, FRQ oscillations, both in amount and phosphorylation state, were absent in the csn-2 mutant (Fig. 3B,C). In the csn-2 mutant, FRQ remained hyperphosphorylated at all time points, and its levels were higher than those of the wild-type strain. These data indicate that FRQ oscillations are abolished when nutrients are limiting in the csn-2 mutant, but weak FRQ rhythms can still persist when there is active protein synthesis.

\section{Abnormal conidiation rhythms of the csn-2 mutant in $D D, L D, L L$, and temperature cycles}

We next examined the circadian conidiation rhythm of the $\operatorname{csn}-2$ mutant by race tube assays. In $\mathrm{DD}$, wild-type strains exhibit robust circadian conidiation rhythm with a period of $\sim 22 \mathrm{~h}$ (Fig. 4A). The csn-2 mutant grew more slowly and produced fewer aerial hyphae and conidia on
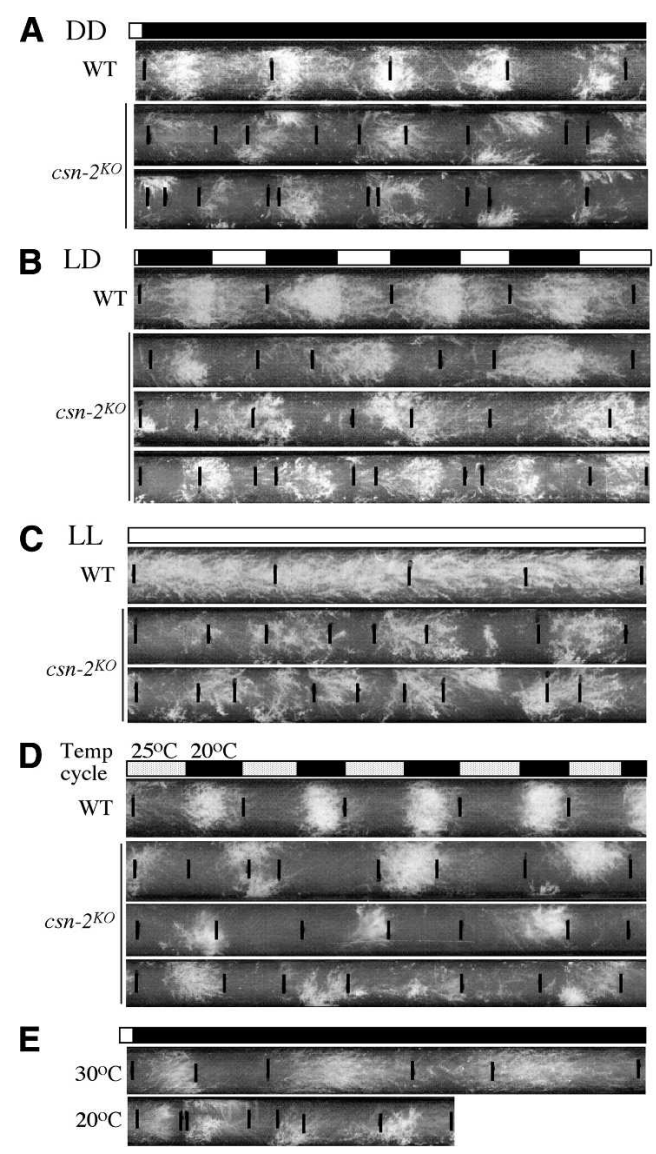

Figure 4. Abnormal conidiation rhythms of the csn-2 mutant in $\operatorname{DD}(A), \operatorname{LD}(B), \operatorname{LL}(C)$, and temperature cycles $(D)$, or at different constant temperatures in DD $(E)$. At least six replicates were examined under each condition and representative race tube results are shown. The black lines mark the growth fronts every $24 \mathrm{~h}$. 
race tubes than the wild type, and its growth rate sometimes varied significantly from day to day. In contrast to the robust and precise timing of conidiation of the wildtype strain, the csn-2 mutant showed an abnormal conidiation pattern in DD in most of the $c s n-2$ race tubes. The duration of two adjacent conidiation bands of the csn-2 strain was occasionally close to $1 \mathrm{~d}$ (the last $2 \mathrm{~d}$ in the bottom race tube in Fig. 4A), but in most cases, the duration was $\sim 2 \mathrm{~d}$. Because of the variability of the mutant growth rate, an accurate period length of this banding pattern could not be determined. These data suggest that the normal circadian conidiation rhythm and circadian clock function were severely impaired in the csn-2 mutant. However, since the abnormal banding pattern in the mutant was observed in most race tubes (from $>20$ race tubes) with a similar duration, it is likely to due to a rhythm of conidiation rather than its random fluctuations.

We have shown previously that, despite the arrhythmic conidiation and abnormal banding patterns in DD in the $f_{w d-1}{ }^{R I P}$ strain, its conidiation rhythm can be entrained by LD cycles (He et al. 2003). Since FRQ expression can respond to LD transition in the csn-2 mutant (Figs. 2, 3), it should be rhythmic under LD cycles. We thus examined the conidiation rhythm of the csn-2 strain in LD cycles (12 h light/12 h darkness). Surprisingly, unlike the wild-type strain, the LD cycles failed to entrain the conidiation rhythms in the csn-2 mutant (Fig. 4B). As in DD, the period length between two major adjacent conidiation peaks was $\sim 2 \mathrm{~d}$ in most cases. In addition, the timing of conidiation is inconsistent from tube to tube, with conidiation sometimes peaking during the light period (middle race tube, Fig. 4B). Thus, unlike the $f_{W d-1}{ }^{R I P}$ strain, the conidiation of the $c s n-2$ mutant cannot be driven by LD cycles, suggesting that the regulation of conidiation by light is impaired in the csn-2 mutant. However, this failure is neither due to a defect in the initial light-signaling pathway nor due to the absence of FRQ oscillation. Thus, the conidiation rhythm of the mutant is likely driven by a FRQ-independent oscillator (or FRQ-less oscillator, FLO) (Iwasaki and Dunlap 2000). Therefore, CSN appears to have a conserved role in regulating light-regulated developmental processes in plants, Aspergillus, Neurospora, and possibly other eukaryotic organisms (Busch et al. 2003; Wei and Deng 2003).

Constant light condition (LL) results in the constant elevation of FRQ expression and constitutive conidiation for the wild-type strain (Fig. 4C) (Crosthwaite et al. 1995; Collett et al. 2002). Although the conidiation of the csn-2 mutant in LL became less organized than that in DD or LD, abnormal banding patterns could still be observed and the period varied from $\sim 1$ to $2 \mathrm{~d}$.

We then asked whether the conidiation rhythm of the csn-2 mutant could be synchronized by temperature cycles. For $f r q$ and $w c$ mutants, although their conidiation processes are arrhythmic in DD and cannot be entrained by LD cycles, their conidiation rhythms can be driven by moderate temperature cycles (Merrow et al. 1999; He et al. 2002; Pregueiro et al. 2005). Under 12 h $25^{\circ} \mathrm{C} / 12 \mathrm{~h} 20^{\circ} \mathrm{C}$ temperature cycles, the conidiation rhythm of the wild-type strain was synchronized, with conidiation occurring in the cold phase (Fig. 4D; Liu et al. 1998). Surprisingly, for the csn-2 mutant, although $\sim 1$-d weak conidiation rhythms were transiently observed in some race tubes for a period (bottom race tube, Fig. 4D), robust $\sim 2$-d conidiation rhythms were seen for most race tubes. These data suggest that, unlike other Neurospora circadian clock mutants, the temperatureregulated conidiation process is also affected in the csn-2 mutant.

We then asked whether the abnormal conidiation rhythm of the $\operatorname{csn}-2$ mutant is temperature compensated in DD (Fig. 4E), a property that is lost in most known FLOs (Dunlap and Loros 2004; Pregueiro et al. 2005). As shown in Figure 4E, the 2-d conidiation rhythm was maintained in $20^{\circ} \mathrm{C}$ and $30^{\circ} \mathrm{C}$ in $\mathrm{DD}$, suggesting that the abnormal rhythm of the $c s n-2^{K O}$ strain may be temperature compensated.

\section{Autoubiquitination of FWD-1 reduces its stability and levels in the csn-2 mutant}

Because the impaired FRQ degradation in the csn-2 mutant is similar to that of the $f_{w d}-1^{R I P}$ strain and because CSN regulates the activity of the SCF complex by removing Nedd8 from cullins, we tested whether the expression level of FWD-1 was affected in the csn-2 mutant. We first generated a FWD-1-specific polyclonal antibody. Consistent with the predicted size of the FWD-1 protein, our FWD-1 antibody specifically recognized a $110-\mathrm{kDa}$ band in the wild-type strain, but not in the $f_{W d-1}$ mutant (Fig. 5A). Compared with the wild-type strain, FWD-1 levels were dramatically lower in the csn$2^{K O}$ mutant strain (Fig. 5B). Moreover, while FWD-1 levels in the mutant could be detected in cultures grown for $2 \mathrm{~d}$, it became undetectable in the 3-d-old cultures. Since FWD-1 is required for the proper degradation of FRQ, these results suggest that the impaired FRQ degradation in the csn-2 mutant is due to low levels of FWD-1. In addition, the steady-state levels of FWD-1 in the mutant are dependent on active protein synthesis as nutrient depletion decreases FWD-1 levels.

Northern blot analysis showed that the level of fwd-1 mRNA in the csn-2 mutant was comparable to that of the wild-type strain (Fig. 5C), suggesting that the regulation of FWD-1 by CSN is not at the level of transcription. To examine whether the low FWD-1 levels in the csn-2 mutant is due to an increased FWD-1 degradation rate, we compared the FWD-1 degradation rates between the wild-type and $c s n-2^{K O}$ strains. As shown in Figure $5 \mathrm{D}, \mathrm{FWD}-1$ was quite stable in the wild-type strain, as a majority of FWD-1 was still present after $12 \mathrm{~h}$ of CHX incubation. In contrast, FWD-1 became undetectable after only $3 \mathrm{~h}$ of CHX treatment in the csn-2 mutant, indicating that it was very unstable. To confirm this result and to quantify the degradation rate of FWD-1 in the csn-2 mutant, we introduced a c-Myc-tagged FWD-1 expressing construct (He et al. 2003) into a csn-2 mutant $\left(\operatorname{csn}-2^{K O}\right.$, Myc-FWD-1). As shown in Figure 5E, like the 


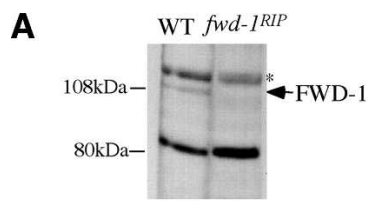

B
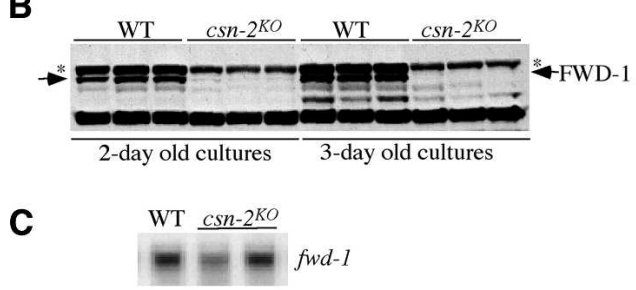

D

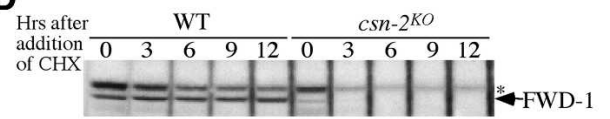

E
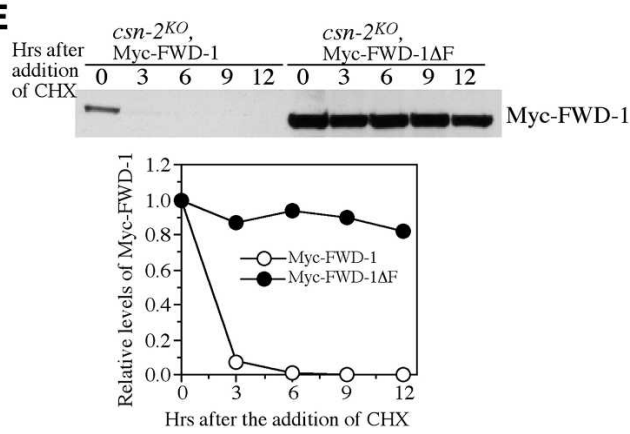

Figure 5. The FWD-1 levels are drastically reduced in the csn-2 mutant, probably due to its rapid autoubiquitination-mediated degradation. (A) Western blot analysis showing the expression of FWD-1 in the wild-type and $f_{w} d-1^{R I P}$ strains. The arrow indicates the FWD-1 specific band in the wild-type strain. The asterisk indicates an unspecific cross-reacted protein band recognized by our FWD-1 antiserum. (B) The expression of FWD-1 in the wild-type and $c s n-2^{K O}$ strains after 2 or $3 \mathrm{~d}$ of growth in liquid medium. $(C)$ Northern blot analysis showing the expression of $f_{w d-1}$ mRNA in the wild-type and $c s n-2^{K O}$ strains. $(D)$ Western blot analysis showing the degradation of FWD-1 after the addition of CHX. Similar results were obtained in three independent experiments. (E) Degradation of Myc-FWD-1 or Myc-FWD-1 $\Delta \mathrm{F}$ (lacking its F-box) expressed in the csn-2 mutants after the addition of CHX. The densitometric analysis of the results is shown in the bottom panel.

endogenous FWD-1, Myc-FWD-1 was very unstable in the csn-2 mutant, with an estimated half-life of $\sim 45 \mathrm{~min}$. Thus, FWD- 1 is degraded $>10$-fold faster in the csn- 2 mutant than in the wild-type strain.

FBPs are known to autoubiquitinate /Cope and Deshaies 2003); thus, the accelerated FWD-1 degradation rate in the csn-2 mutant is likely due to its increased autoubiquitination. If so, the removal of the F-box domain from FWD-1 should disrupt the formation of the SCF complex and prevent its autoubiquitination and degradation. Indeed, the Myc-tagged FWD-1 lacking its F-box (Myc-FWD-1 $\Delta \mathrm{F})$ (He et al. 2003) was very stable and accumulated to high steady-state levels in the csn-2 mutant (Fig. 5E). These data are consistent with the notion that the instability of FWD-1 in the csn-2 mutant is caused by its autoubiquitination.

Together, these data indicate that CSN is important for maintaining the stability of FWD-1 in Neurospora, probably by preventing its autoubiquitination. Although FWD-1 levels are low in the csn-2 mutant, some active SCF complexes can still form and mediate the degradation of its substrates, such as FRQ. Since FWD-1 is very unstable in the csn-2 mutant, the maintenance of a functional SCF complex relies mostly on the newly synthesized FWD-1 protein. When protein synthesis is blocked by $\mathrm{CHX}$ or slowed down by nutrient depletion, levels of functional $\mathrm{SCF}^{\mathrm{FWD}-1}$ complex further decrease. These data explain the nutrient-dependent FRQ oscillations of the csn-2 strain shown in Figure 3 and at least in part resolve the CSN paradox in Neurospora.

\section{Identification of the components in the SCF $F^{F D D-1}$ complex}

Since FWD-1 is very unstable in the $\operatorname{csn}$-2 mutant, we asked whether the entire $\mathrm{SCF}^{\mathrm{FWD}-1}$ complex is regulated in a similar manner. To address this, we identified the other components in the $\mathrm{SCF}^{\mathrm{FWD}-1}$ complex by biochemical purification. We introduced a Myc-His-tagged FWD-1 construct (FWD-1 was tagged by a 5-c-Myc tag and a 6 -His tag at the $\mathrm{N}$ terminus, and the fusion protein is under the control of the $q a-2$ promoter) into the fwd$1^{R I P}$ strain. The Myc-His-FWD-1 was able to rescue the circadian defects of the $f_{w d}-1^{R I P}$ strain (Fig. 6A), indicating that the tagged FWD-1 was functional. Myc-HisFWD-1 was purified by nickel column followed by immunoprecipitation using a Myc monoclonal antibody. Two major specific bands at 120 and $19 \mathrm{kDa}$ with similar molar ratios were observed in the Myc-His-FWD-1 sample, but not in the sample prepared from a wild-type strain lacking the epitope-tagged FWD-1 (Fig. 6B). Protein identification by mass spectrometry identified the top band as Myc-His-FWD-1 and bottom band as the Neurospora homolog of SKP-1 (also called SCON-3, referred to as SKP-1 hereafter to follow the nomenclature for SCF components) (Sizemore and Paietta 2002).

There were several additional minor bands with sizes ranging from 75 to $120 \mathrm{kDa}$ in the Myc-His-FWD-1 immunoprecipitates (IPs). In an SCF complex, SKP-1 bridges the interaction between the FBP and cullin subunits. The Neurospora genome encodes three cullin-like proteins with predicted protein molecular masses ranging from 85 to $110 \mathrm{kDa}$. However, mass spectrometry analysis of the minor bands only identified degradation products of the Myc-His-FWD-1 protein, which could mask the presence of substoichiometric amounts of cullins in the IP. Regardless, the cullin and RING subunits are not present in the complex in stoichiometric amounts, and most of the FWD-1/SKP-1 heterodimer is not associated with the cullin/RING subunits.

Since CUL-1 homologs are known to be the cullin subunit in eukaryotic SCF complexes (Petroski and Deshaies 2005), we next examined whether the Neurospora 
He et al.

A
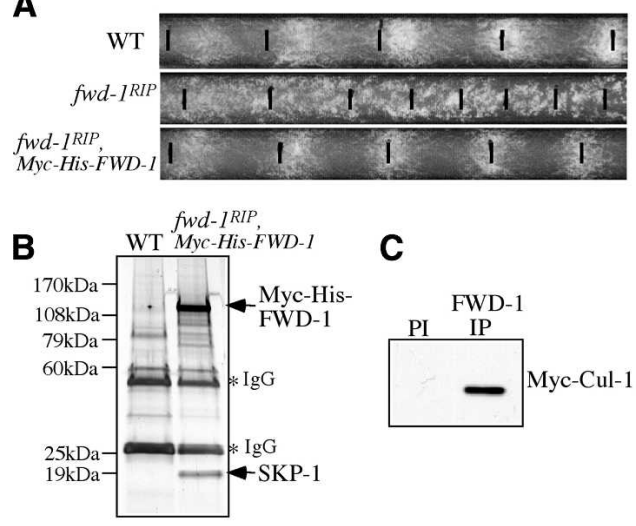

D

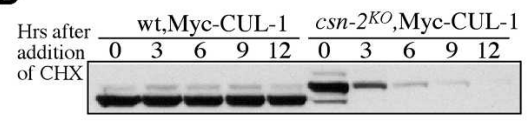

$\mathbf{E}$

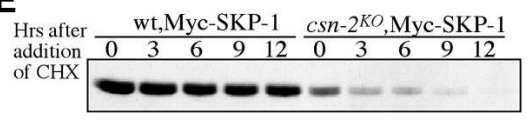

$\mathbf{F}$
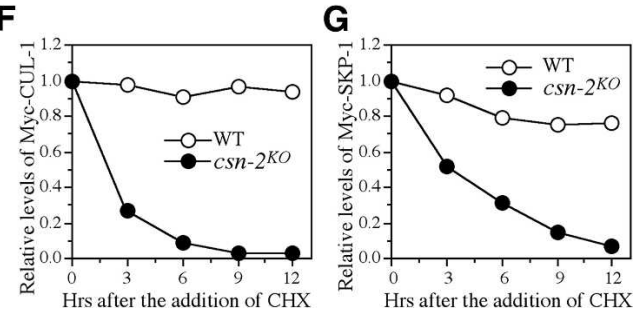

Figure 6. The components of the $\mathrm{SCF}^{\mathrm{FWD}-1}$ complex, SKP1 and CUL-1, are also unstable in the csn-2 mutant. (A) Race tube assays showing the complementation of the circadian conidiation rhythm defect of the fwd-1 ${ }^{R I P}$ strain by the expression Myc-His-FWD-1. (B) Silver-stained SDS-PAGE gel showing biochemical purification of Myc-His-FWD-1 and its associated proteins. A wild-type strain was used as the negative control. MycHis-FWD-1 and SKP-1 were identified by mass spectrometry analysis. $(C)$ Immunoprecipitation (IP) assays showing the association between FWD-1 and Myc-CUL-1. The extracts of the wt,Myc-Cul-1 strain were immunoprecipitated by the preimmune serum (PI) or the FWD-1 antiserum, followed by Western blot analysis using the c-Myc antibody. $(D, E)$ Degradation of Myc-CUL-1 $(D)$ or Myc-SKP-1 $(E)$ in the wild-type strain or the csn-2 mutant. Similar results were obtained in two independent experiments. The densitometric analyses of the results in $D$ and $E$ are shown in $F$ and $G$, respectively.

CUL-1 form a complex with FWD-1 in vivo by IP-Western experiments. Myc-CUL-1 was specifically IPed by the FWD-1 antibody, suggesting that CUL-1 is a component of the $\mathrm{SCF}^{\mathrm{FWD}-1}$ complex (Fig. 6C). The RING subunit of the SCF complex is an RBX1-like protein, which recruits the E2 enzyme for ubiquitination. There is only one RBX1-like protein in Neurospora (NCU06224.1). Thus, it should be the RING subunit of the $\mathrm{SCF}^{\mathrm{FWD}-1}$ complex. Interestingly, at the amino acid level, the putative Neurospora RBX-1 protein is $94 \%$ identical to human RBX1, while it is only $63 \%$ identical to Hrtlp in $S$. cerevisiae.
SKP-1 and CUL-1 are unstable in the csn-2 mutant

Having identified SKP-1 and CUL-1 as the components of the $\mathrm{SCF}^{\mathrm{FWD}-1}$ complex, we then examined whether the stability of Myc-SKP-1 or Myc-CUL-1 is affected in the $c s n-2$ mutant. As shown in Figure $6 \mathrm{D}-\mathrm{G}$, both MycSKP-1 and Myc-CUL-1 were very stable in the wild-type strain, with a half-life $>12 \mathrm{~h}$ in the presence of CHX. In contrast, both proteins became unstable in the csn-2 mutant, with estimated half-lives of $3 \mathrm{~h}$ and $1.5 \mathrm{~h}$, respectively. Because autoubiquitination is likely responsible for the fast degradation of FWD-1 in the csn-2 mutant, the accelerated Myc-CUL-1 and Myc-SKP-1 degradation in the mutant may be also due to their autoubiquitination. Therefore, CSN is important for maintaining the stability of the entire SCF ${ }^{\mathrm{FWD}-1}$ complex in Neurospora.

SCON-2, another F-box/WD40 repeat containing protein, is also very unstable in the csn-2 mutant

We then asked whether CSN also regulates other SCF complexes in a similar manner. The Neurospora SKP-1 protein was previously shown to interact with SCON-2, an F-box/WD40 repeat-containing protein involved in regulating sulfur metabolism (Sizemore and Paietta 2002). We compared the expression of Myc-SCON-2 in the wild-type and csn-2 mutant strains. The levels of Myc-SCON-2 in the csn-2 mutant were dramatically lower than those in the wild-type strain (Fig. 7A). The half-life of Myc-SCON-2 was $\sim 6-9$ h in the wild-type strain in the presence of CHX. As expected, MycSCON-2 was very unstable in the csn-2 mutant and became undetectable after only $3 \mathrm{~h}$ of CHX treatment (Fig. 7B). Therefore, the CSN is also important for maintaining the stability of other F-box containing adapter proteins.

\section{Purification and identification of the components of the Neurospora CSN}

CSN in most eukaryotes contains eight subunits (CSN18). Six CSN subunits contain the PCI domain, while two subunits have an MPN (Mpr1-Pad1-N-terminal) domain (Deng et al. 2000; Li and Deng 2003; Wei and Deng 2003). To confirm that the Neurospora CSN is similar to other eukaryotic CSNs and to examine whether most of CSN-2 is part of the CSN or it forms subcomplexes with other proteins, we decided to purify the CSN-2 associated protein from Neurospora. To do so, we first introduced a Myc-His-tagged CSN-2 expressing construct (similar to the Myc-His-FWD-1 construct) into a csn-2 mutant. The expression of Myc-His-CSN-2 rescued the circadian clock defect of the csn-2 mutant, resulting in a robust daily conidiation rhythm similar to the wild-type strain (Fig. 7C). This indicates that the Myc-His-CSN-2 fusion protein is functional. Myc-His-CSN-2 was purified by nickel-column followed by immunoprecipitation using c-Myc monoclonal antibody. A wild-type strain was used as the negative control. As shown in Figure 7D, several major protein bands were only observed in the Myc-His-CSN-2 sample. These bands were excised for 
A

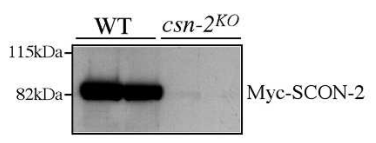

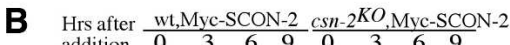
addition 0 \begin{tabular}{llllllll}
\cline { 2 - 5 } & 0 & 6 & 9 & 0 & 3 & 6 & 9
\end{tabular} of $\mathrm{CHX}$
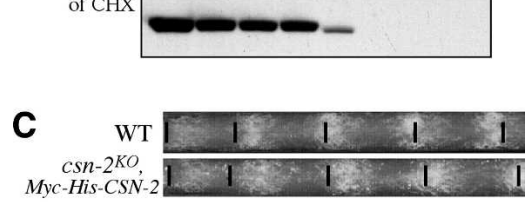

D

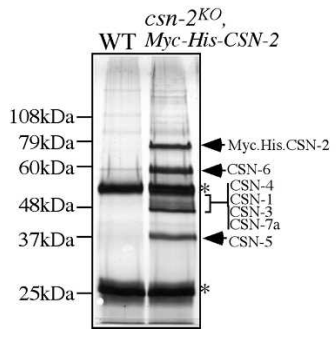

Figure 7. SCON-2 is rapidly degraded in the $\operatorname{csn}-2$ mutant, resulting in its drastically reduced expression level. $(A)$ Expression level of Myc-SCON-2 in the wild-type and $c s n-2^{K O}$ strains in LL. Two independent transformants of each strain were used. (B) Western blot analysis showing the degradation of MycSCON-2 in the wild-type and $c s n-2^{K O}$ strains. $(C)$ Race tube assays showing the complementation of the circadian conidiation rhythm defect of the $c n s-2^{K O}$ strain by the expression MycHis-CSN-2. (D) Silver-stained SDS-PAGE gel showing biochemical purification of the Neurospora CSN. A wild-type strain was used as the negative control. CSN subunits identified by mass spectrometry analysis are indicated. Asterisks indicate the two IgG bands.

mass spectrometry analysis, which led to the identification of seven proteins. In addition to the Myc-His-CSN2, these proteins are all predicted Neurospora homologs of the CSN subunits, from CSN-1 to CSN-7a, according to the unified nomenclature for CSN components (CSN-1, NCU00157.1; CSN-3, NCU01408.1; CSN-4, NCU07361.1; CSN-5, NCU00467.1; CSN-6, NCU07019.1; and CSN-7a, NCU08342.1). Neurospora has another csn-7-like gene in the genome (csn-7b, NCU02813.1), but it was not identified in the purification products. It either does not form a stable complex with CSN or its level is too low. No CSN-8-like protein was identified, consistent with the absence of a csn-8-like gene in the Neurospora genome and with the fact that CSN8 is the least conserved CSN subunit. No other protein was present in significant amounts in the purification products. These data indicate that the Neurospora CSN is similar to other eukaryotic CSNs and most, if not all, of the CSN-2 is in the CSN and not in other subcomplexes, further suggesting that the phenotypes we observed in the $\operatorname{csn}-2$ mutant is due to the loss of CSN function.

\section{Discussion}

CSN is an evolutionarily conserved multifunctional protein complex found in eukaryotes. We show here that
CSN is an important component of the Neurospora circadian clock by regulating FRQ degradation. In addition to circadian clock defects, light and temperature entrainment of conidiation are impaired in the $c s n-2$ mutant. In the csn-2 mutant, a FLO regulates its conidiation, resulting in abnormal conidiation rhythms. Furthermore, we showed that CSN is important for maintaining the stability of the SCF complexes in Neurospora, explaining the CSN paradox in this organism.

\section{The roles of CSN in the Neurospora circadian clock}

Regulation of clock protein degradation is an important mechanism controlling circadian clocks in eukaryotes. The post-translational regulation of FRQ and PER and their degradation process are conserved from Neurospora to animals. In the csn-2 mutant, FRQ degradation is impaired, especially when protein synthesis is blocked. In DD, FRQ oscillations are abolished or severely compromised in the mutant. The csn-2 mutant exhibits abnormal conidiation rhythms in DD, with a period of $\sim 2 \mathrm{~d}$. Surprisingly, such an abnormal rhythm of the mutant cannot even be entrained by LD and temperature cycles, suggesting that the light and temperature regulations of the conidiation process are impaired in the mutant. These data suggest that CSN is an important regulator of the circadian clock in Neurospora. We further show that defective FRQ degradation in the csn-2 mutant is due to drastically reduced stability and levels of FWD-1. Thus, low levels of SCF ${ }^{\mathrm{FWD}-1}$ complex, especially under slowgrowth conditions, lead to accumulation of FRQ and impaired circadian rhythms. Because of the conservation of the degradation pathway of PER and FRQ from Neurospora to animals, CSN is likely to be another conserved component of the eukaryotic circadian clocks.

\section{The Smith-Magenis syndrome}

The Smith-Magenis syndrome (SMS) is a multiple congenital anomalies/mental retardation syndrome arising from a hemizygous deletion of human chromosome 17, band p11.2 (Greenberg et al. 1996; Potocki et al. 2000; De Leersnyder et al. 2001). The deleted region spans 1.5-2.0 $\mathrm{Mb}$ of DNA, which contains $\sim 20$ genes. The clinical phenotype includes craniofacial abnormalities, developmental anomalies, development delay, mental retardation, and abnormal behavior. In addition, sleep disturbances (such as early sleep onset and early waking) and a phase shift of the circadian rhythms of melatonin were found in patients with this disease (Greenberg et al. 1996; Smith et al. 1998; Potocki et al. 2000; De Leersnyder et al. 2001), suggesting that they have an aberrant biological clock. The actual gene(s) causing these phenotypes are not known. Interestingly, all patients with the common deletion are haploinsufficient for the CSN3 (COPS3) subunit of the human CSN, which was mapped to the distal portion of the SMS critical interval (Elsea et al. 1999; Potocki et al. 1999, 2000). The evolutionary conservation of CSN functions in eukaryotes and the 
He et al.

conservation of post-translational regulations of circadian clock between Neurospora and animals raises the possibility that a partial deficiency in CSN function might contribute to the circadian and sleep disturbance phenotypes in SMS patients.

\section{Abnormal conidiation rhythms in the csn-2 mutant}

Although the conidiation rhythm of the csn-2 mutant cannot be entrained by LD 12/12 and temperature cycles, FRQ expression is still regulated by the light/dark cycles, as indicated by the decrease of FRQ levels after LD transition. Thus, FRQ level should be rhythmic under LD cycles. Furthermore, the abnormal conidiation rhythm of the mutant was observed in LL, a condition leading to constant elevation of FRQ expression. Thus, the abnormal conidiation rhythm of the csn-2 mutant is most likely due to the uncoupling of the FRQ oscillation from the conidiation process. In other words, it is an oscillation driven by a FLO (Iwasaki and Dunlap 2000; Dunlap and Loros 2004). The differences in race tube phenotypes between the csn-2 and fwd-1 mutants suggest that the conidiation rhythm of the csn-2 strain is due to its influence on another cullin-containing ubiquitin ligase in Neurospora, which leads to the uncoupling of the conidiation process from the fr $q$ - $w c$ oscillator and the light and temperature signaling pathways.

Several FLOs, mostly found using conidiation as the output, have been described in $f r q$ or $w c$ null mutant strains (Loros and Feldman 1986; Aronson et al. 1994b; Merrow et al. 1999; Lakin-Thomas and Brody 2000; Correa et al. 2003; Granshaw et al. 2003; Christensen et al. 2004; Dunlap and Loros 2004). Most of these oscillations do not appear to be circadian because of their highly variable period length, the inability to be entrained by light, and the loss of temperature and nutritional compensation. A recent study showed that one of the FLOs visualized under temperature cycles (Merrow et al. 1999) is a slave oscillator controlled by the fr $q$-wc-based circadian transcription and translation feedback loop and is not the underlying circadian-rhythm generator as proposed previously (Pregueiro et al. 2005). Different from these known FLOs, the FLO in the csn-2 mutant appears to be temperature compensated. Thus, FRQ may not be required for a temperature-compensated conidiation rhythm in Neurospora. However, we currently do not know whether the FLO in the csn-2 mutant contributes to the circadian clock function in a wild-type strain or whether it only exists in the absence of a functional frqwC oscillator.

Resolving the CSN paradox: the role of CSN in regulating the stability, recycling, and assembly of cullin-based ubiquitin ligases

The known biochemical activity of CSN is opposite from its known in vivo functions, giving rise to a CSN paradox (Cope and Deshaies 2003; Wei and Deng 2003; Wolf et al. 2003). To reconcile this paradox, CSN has been proposed to be a key regulator of the assembly/ disassembly and maintenance platform for the SCF-type ubiquitin ligases. In this model, the CSN-mediated cullin deneddylation allows the dissociation of the cullin from the FBP and the formation of the CUL1-CAND1 complex. CAND1 preferentially forms a complex with unneddylated cullins (Liu et al. 2002; Zheng et al. 2002; Feng et al. 2004; Goldenberg et al. 2004) and has a homolog in Neurospora (NCU08875.1). Thus, the neddylation/deneddylation of cullins is critical for the SCF cycle.

Our results presented above provide strong experimental support for this model and an explanation for the CSN paradox in Neurospora (Fig. 8). Like CSN in other eukaryotic systems, the Neurospora CSN is required for the deneddylation of CUL-1 and is important for the degradation of FRQ, an $\mathrm{SCF}^{\mathrm{FWD}-1}$ substrate. Our results indicate that CSN is important for maintaining the stability of SCF complexes. Figure 8 is a model of the SCF cycle based on previous CSN studies and the results of this work. In a strain with a functional CSN, activated SCF complexes will be disassembled after deneddylation of the cullin subunits. The unneddylated cullin will then form a complex with CAND-1 (Fig. 8A). This process inactivates the ligase complex and separates the SKP-1FBP from the rest of the complex, preventing their autoubiquitination and destruction. Once cullin becomes neddylated again by the neddylation enzyme, the SKP1-FBP complex replaces CAND-1 and reforms an active SCF complex, completing an SCF assembly/disassembly cycle. Thus, in a wild-type strain, activated SCF com-

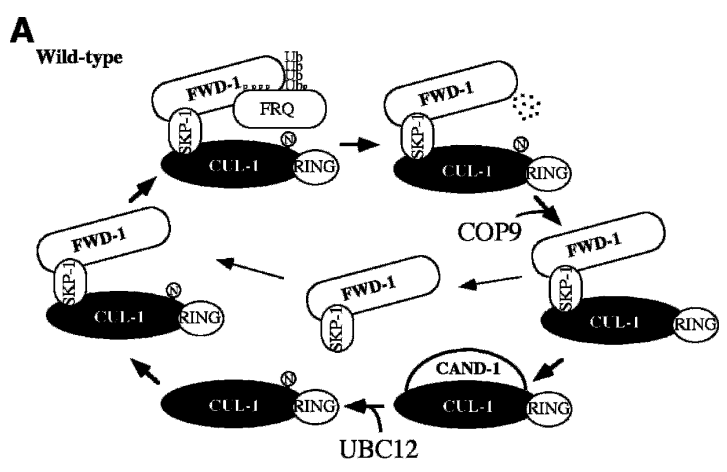

B

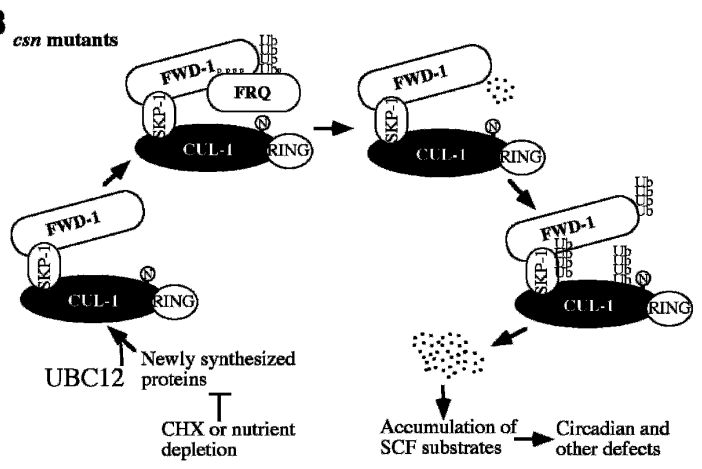

Figure 8. A model of the $\mathrm{SCF}^{\mathrm{FWD}-1}$ cycle in a wild-type $(A)$ strain and in csn mutants $(B)$. 
plexes only form transiently and are quickly disassembled and inactivated by CSN-mediated cullin deneddylation, and the components of the SCF complexes are stable. This conclusion is supported by several lines of evidence. First, most of CUL-1 exists in its unneddylated state in a wild type (Fig. 1C), suggesting that most of CUL-1 is presumably not in complex with SKP-1/FBP. Second, purification of FWD-1 showed that most of the SKP-1/FWD-1 complexes are not associated with CUL-1. The presence of CUL-1 in the complex can only be detected by Western blot. Third, the components of the SCF complexes, including FWD-1, SCON-2, SKP-1, and CUL-1, are all very stable (half-life $>6 \mathrm{~h}$ ) in a wild-type strain despite their presence in the ubiquitin ligase complexes (Figs. 5-7).

In contrast to the wild-type strain, such an SCF assembly/disassembly cycle is disrupted in mutants lacking a functional CSN (Fig. 8B). In csn mutants, active SCF complexes can still form from the newly synthesized proteins and are still capable of mediating substrate degradation, as indicated by the degradation of FRQ after a LD transition (Fig. 5). However, after degradation of their substrates, the active SCF complexes cannot be disassembled because CUL-1 stays neddylated. Thus, CAND1 cannot replace SKP-1-FBP to form a complex with CUL-1 to inactivate the ligase complex, leading to the autoubiquitination and destruction of the SCF components. Consistent with this notion, a FWD-1 mutant protein that lacks its F-box and cannot form a complex with SKP-1 is very stable and accumulates to high levels in the csn-2 mutant (Fig. 5). The rapid degradation of FBPs and other SCF components leads to their low levels in csn mutants, resulting in the accumulation of their substrates, such as FRQ, causing circadian and other defects. Therefore, the main defect of the csn mutants appears to be the lack of sufficient amounts of cullin ubiquitin ligases.

Although CSN is important for the stability, recycling, and assembly/disassembly of SCF complexes, it does not appear to be required for SCF biochemical activities. On the contrary, the lack of cullin deneddylation should stimulate SCF activity. However, in csn mutants, active SCF complexes and degradation of their substrates are largely dependent on newly synthesized SCF components. When protein synthesis is slowed down by nutrient depletion or blocked by protein synthesis inhibitor, the levels of the FBPs become further reduced and substrate degradation is further impaired (Figs. 2, 3, 5). Thus, if protein synthesis is able to provide sufficient amounts of SCF components, the defects in protein degradation can be overcome in csn mutants. Consistent with this, the disruption of csn 4 or csn 5 in fission yeast Schizosaccharomyce pombe, although leading to hyperneddylation of Pculand Pcu3, has no obvious physiological phenotypes (Mundt et al. 2002). On the other hand, the deletion mutants of $\operatorname{csn} 1$ and $\operatorname{csn} 2$ in $S$. pombe are delayed in S-phase and sensitive to DNA damage (Mundt et al. 1999|, suggesting that different CSN subunits have different contributions for CSN functions. These latter phenotypes are due to hyperneddylation of Pcu4 and the impaired degradation of Spd1, an inhibitor of ribonucleotide reductase (Liu et al. 2003). Purification of Csn2-TAP in $S$. pombe showed that Ddb1 and Pcu4 are present in stoichiometric amounts with the CSN (Liu et al. 2003). The absence of stable association of these proteins with the Neurospora CSN suggests that the role and regulation of CSN in Neurospora might be different from $S$. pombe.

The stability of FBP Poplwas modestly decreased in the $\operatorname{csn} 5 \Delta$ mutant in $S$. pombe (Zhou et al. 2003). However, its destabilization is largely attributed to the CSNassociated Ubp12, a deubiquitinating enzyme, and it was proposed that both the CSN and the CSN-associated Ubp12 work together to prevent autoubiquitination of SCF complexes. Very recently, a study by Wee et al. (2005) confirmed this and showed that Btb3p, the Cul3p adapter protein, was present at low levels and was degraded faster in the $c s n$ and $u b p 12$ mutants. In the $\operatorname{csn} 5$ or ubp12 mutants, the half-life of Btb3p was reduced from $60 \mathrm{~min}$ to 40 and $20 \mathrm{~min}$, respectively, so that the effect on Btb3p stability is more severe in the ubp12 mutant than in the cns-5 mutant. However, the disruption of ubp12 in S. pombe, like that of csn-4 or csn5, has no obvious physiological phenotype.

Only one ubp12-like gene exists in Neurospora (NCU00480.1), but its disruption has no detectable phenotype in growth, clock function, FWD-1 level, and FRQ degradation (Q. He and Y. Liu, unpubl.). Thus, it appears that UBP-12 does not contribute significantly in maintaining the stability of SCF complexes in Neurospora, although it is possible that another protein replaced its function in the ubp-12 mutant.

The FBPs of Neurospora are long-lived in Neurospora (half-life $>6$ h) (Figs. 5-7). In contrast, the FBPs of budding and fission yeast are very unstable (half-life 5-60 min) (Zhou and Howley 1998; Zhou et al. 2003). In this respect, the Neurospora FBPs are similar to those in animals (Davis et al. 2002; Cope and Deshaies 2003). In yeast, the rapid degradation of the FBPs should require active protein synthesis of SCF components to maintain their appropriate levels for cellular functions. Thus, although CSN can modestly affect FBPs' stability in yeast, the lack of CSN is overcome by active protein synthesis of FBPs. This may explain the lack of phenotype in the csn and ubp12 mutants in S. pombe. On the other hand, in organisms such as Neurospora, in which FBPs are relatively long-lived and presumably are not actively synthesized, the regulation of the stability and recycling of SCF by CSN becomes critical. In the case of FWD-1, its halflife drops from $>6 \mathrm{~h}$ in the wild-type strain to $\sim 45 \mathrm{~min}$ in the csn-2 mutant. In addition, CUL-1 and SKP-1 also become unstable in the csn-2 mutant. Together, these events lead to low levels of SCF ${ }^{\mathrm{FWD}-1}$ complex and accumulation of its substrates.

In conclusion, the results presented here show that the Neurospora CSN regulates the circadian clock by controlling the stability of the $\mathrm{SCF}^{\mathrm{FWD}-1}$ complex. In addition, we showed that an FRQ-independent oscillator drives conidiation rhythm in the csn-2 mutant. Furthermore, our data provide an explanation to the CSN para- 
dox. Because CSN is important for SCF functions in higher eukaryotes, we propose that CSN may have a similar role in those organisms as well.

\section{Materials and methods}

\section{Strains and culture conditions}

The $b d, a$ (wild-type clock) strain was used as the wild-type strain in this study. The $b d, f_{w d-1} 1^{R I P}$ and $b d$, fwd-1 $1^{R I P}$, his-3 strains generated previously were also used in this study (He et al. 2003). The $b d, c s n-2^{K O}$ and $b d, c s n-2^{K O}$, his-3 strains were created in this study. The 301-6 (bd, his-3, A) strain, the $b d$, fwd-1 ${ }^{R I P}$, his-3 strain, and the $b d, c s n-2^{K O}$, his-3 strain were the host strains for the his-3 targeting constructs. Liquid culture conditions were the same as described previously unless otherwise noted (Aronson et al. 1994a). For liquid cultures containing QA, 0.01 M QA (pH 5.8) were added into liquid culture medium containing $1 \times$ Vogel's, $0.1 \%$ glucose, and $0.17 \%$ arginine /Cheng et al. 2001b). Race tube assay medium contained $1 \times$ Vogel's, $0.1 \%$ glucose, and $0.17 \%$ arginine, $50 \mathrm{ng} / \mathrm{mL}$ biotin, and $1.5 \%$ agar. For race tubes containing QA $\left(10^{-3} \mathrm{M}\right)$, no glucose was added into the medium.

\section{Disruption of the csn-2 gene in Neurospora}

The Neurospora csn-2 gene was disrupted by gene replacement. A PCR fragment containing the entire CSN-2 ORF and its 3 'UTR was cloned into pDE3dBH, resulting in pCSN-2. To make the disruption construct, a hph fragment was inserted into the BamHI site of pCSN-2. A PCR fragment containing the gene replacement cassette was introduced into 87-3 (bd, a) (wildtype) strain by electroporation. PCR analysis was performed to identify transformants that carried the insertion of $h p h$ at the csn-2 locus, and a positive transformant was crossed with a wild-type strain $(b d$, his-3, A). Sexual spores of the cross were picked individually and germinated on slants containing hygromycin. Southern blot analysis was used to confirm the $c s n-2^{K O}$ strains. A $b d, c s n-2^{K O}$, his-3 strain was used as the host strain for pqa-Myc-His-CSN-2, pqa-Myc-FWD-1, pqa-Myc-FWD-1 $\mathrm{F}$, pqa-Myc-CUL-1, pqa-Myc-His-SKP-1, and pqa-Myc-His-SCON-2 plasmids.

\section{Plasmids}

To create an expression vector that can express Neurospora protein with a Myc-His tag at the $\mathrm{N}$ terminus, a 6-His tag was introduced downstream of the $5 \mathrm{c}-\mathrm{Myc}$ in pqa-5Myc by in vitro mutagenesis (He et al. 2003), resulting in pqa-Myc-His. The fusion proteins are under the control of the quinic acid (QA) inducible qa-2 promoter. To make the his-3 targeting qa-Myc or qa-Myc-His constructs, a PCR fragment containing the entire protein ORF (FWD-1, Cul-1, CSN-2, SKP-1, or SCON-2) and its $3^{\prime}$-UTR was cloned into pqa.Myc-His or pqa-5Myc vectors. The pqa-Myc-FWD-1 and pqa-Myc-FWD $1 \Delta \mathrm{F}$ constructs were created previously (He et al. 2003). The resulting constructs were transformed into appropriate Neurospora strains at the his-3 locus. Positive transformants were confirmed by Western blot analysis using a monoclonal c-Myc antibody (9E10, Santa Cruz Biotechnology).

Purification of the Myc-His-FWD-1 and Myc-His- CSN-2 from Neurospora

The fwd-1 ${ }^{R I P}$, Myc-His-FWD-1, the $c s n-2^{K O}$, Myc-His-CSN-2, and the wild-type strain (as the negative control) were cultured in LL in liquid medium containing QA $10.01 \mathrm{M}$ QA, $1 \times$ Vogel's, $0.1 \%$ glucose, and $0.17 \%$ arginine). Approximately 25 grams of Neurospora tissue from each strain grown in LL was harvested. All purification procedures were carried out at $4^{\circ} \mathrm{C}$. Whole-cell lysate $(400 \mathrm{mg})$ in $100 \mathrm{~mL}$ extraction buffer $(20 \mathrm{mM}$ Tris. Cl at $\mathrm{pH} 7.4,137 \mathrm{mM} \mathrm{NaCl}, 10 \%$ glycerol, and 10-20 mM imidazole plus protease inhibitors) was loaded into a 1-mL Ni-NTA Agarose beads column (QIAGEN). After washing with the washing buffer (20 mM Tris.Cl at $\mathrm{pH} 7.4,300 \mathrm{mM} \mathrm{NaCl}$, and $20 \mathrm{mM}$ imidazole), the bound proteins were eluted by the elution buffer (20 mM Tris.Cl at pH 7.4, $137 \mathrm{mM} \mathrm{NaCl}, 20 \%$ glycerol, and 200 $\mathrm{mM}$ imidazole). After Western blot analysis, fractions containing Myc-His-FWD-1 or Myc-His-CSN-2 were identified. Immunoprecipitation was performed by adding $20 \mu \mathrm{L}$ c-Myc monoclonal antibody-coupled agarose beads (9E10AC, Santa Cruz Biotechnology). After incubation at $4^{\circ} \mathrm{C}$ with gentle rotation for 4-5 $\mathrm{h}$, the beads were washed four times with buffers containing $20 \mathrm{mM}$ Tris. $\mathrm{Cl}(\mathrm{pH} 7.5)$ and $50 \mathrm{mM} \mathrm{NaCl}$, and $20 \mathrm{mM}$ Tris.Cl (pH 7.5) and $500 \mathrm{mM} \mathrm{NaCl}$ before being washed with water once. The precipitates were separated in SDS-PAGE gel and the gel was subsequently silver stained according to the manufacturer's instructions (SiverQuest, Invitrogen). The specific bands were excised and subjected to tryptic digestion and NanoHPLC/electrospray mass spectrometry analysis (University of Texas Southwestern Protein Chemistry Core facility).

\section{Generation of antiserum against FWD-1}

GST-FWD-1 (containing FWD-1 amino acids 168-854) fusion protein was expressed in BL21 cells and the inclusion bodies containing the recombinant protein were purified and used as the antigen to generate rabbit polyclonal antiserum, as described previously (Cheng et al. 2001a). The antiserum was depleted using tissues of the $f w d-1^{R I P}$ strain as previously described (Garceau et al. 1997).

\section{Protein and RNA analyses}

Protein extraction, quantification, Western blot analysis, and immunoprecipitation assay were performed as previously described (Garceau et al. 1997; Cheng et al. 2001a). Immunoprecipitates or equal amounts of total protein $(40 \mu \mathrm{g})$ were loaded in each protein lane. After electrophoresis, proteins were transferred onto PVDF membrane, and Western blot analysis was performed. For Northern blot analysis, equal amounts of total RNA $(20 \mu \mathrm{g})$ were loaded onto agarose gels for electrophoresis, and the gels were blotted and probed with RNA probe specific for fwd-1.

\section{Acknowledgments}

We thank Lixin Wang for excellent technical assistance; Mekhala Maiti for assistance in purification; and Drs. Hongtao $\mathrm{Yu}$, George DeMartino, and James Stull for critical reading and editing of the manuscript. We thank Yue Chen from Southwestern Protein Chemistry Core facility for protein identification. Supported by grants from National Institutes of Health (GM062591 and GM068496) and Welch Foundation to Y.L. Y.L. is the Louise W. Kahn endowed scholar in Biomedical Research at University of Texas Southwestern Medical Center.

\section{References}

Akten, B., Jauch, E., Genova, G.K., Kim, E.Y., Edery, I., Raabe, T., and Jackson, F.R. 2003. A role for CK2 in the Drosophila circadian oscillator. Nat. Neurosci. 6: 251-257. 
Aronson, B., Johnson, K., Loros, J.J., and Dunlap, J.C. 1994a. Negative feedback defining a circadian clock: Autoregulation in the clock gene frequency. Science 263: 1578-1584.

Aronson, B.D., Johnson, K.A., and Dunlap, J.C. 1994b. The circadian clock locus frequency: A single ORF defines period length and temperature compensation. Proc. Natl. Acad. Sci. 91: 7683-7687.

Busch, S., Eckert, S.E., Krappmann, S., and Braus, G.H. 2003. The COP9 signalosome is an essential regulator of development in the filamentous fungus Aspergillus nidulans. Mol. Microbiol. 49: 717-730.

Cheng, P., Yang, Y., Heintzen, C., and Liu, Y. 2001a. Coiled-coil domain mediated FRQ-FRQ interaction is essential for its circadian clock function in Neurospora. EMBO J. 20: 101108.

Cheng, P., Yang, Y., and Liu, Y. 2001b. Interlocked feedback loops contribute to the robustness of the Neurospora circadian clock. Proc. Nat1. Acad. Sci. 98: 7408-7413.

Cheng, P., Yang, Y., Gardner, K.H., and Liu, Y. 2002. PAS domain-mediated WC-1/WC-2 interaction is essential for maintaining the steady state level of WC-1 and the function of both proteins in circadian clock and light responses of Neurospora. Mol. Cell. Biol. 22: 517-524.

Cheng, P., Yang, Y., Wang, L., He, Q., and Liu, Y. 2003. WHITE COLLAR-1, a multifunctional Neurospora protein involved in the circadian feedback loops, light sensing, and transcription repression of wc-2. J. Biol. Chem. 278: 3801-3808.

Cheng, P., He, Q., He, Q., Wang, L., and Liu, Y. 2005. Regulation of the Neurospora circadian clock by an RNA helicase. Genes \& Dev. 19: 234-241.

Christensen, M.K., Falkeid, G., Loros, J.J., Dunlap, J.C., Lillo, C., and Ruoff, P. 2004. A nitrate-induced frq-less oscillator in Neurospora crassa. J. Biol. Rhythms 19: 280-286.

Collett, M.A., Garceau, N., Dunlap, J.C., and Loros, J.J. 2002. Light and clock expression of the Neurospora clock gene frequency is differentially driven by but dependent on WHITE COLLAR-2. Genetics 160: 149-158.

Cope, G.A. and Deshaies, R.J. 2003. COP9 signalosome: A multifunctional regulator of SCF and other cullin-based ubiquitin ligases. Cell 114: 663-671.

Cope, G.A., Suh, G.S., Aravind, L., Schwarz, S.E., Zipursky, S.L., Koonin, E.V., and Deshaies, R.J. 2002. Role of predicted metalloprotease motif of Jab1/Csn5 in cleavage of Nedd8 from Cul1. Science 298: 608-611.

Correa, A., Lewis, Z.A., Greene, A.V., March, I.J., Gomer, R.H., and Bell-Pedersen, D. 2003. Multiple oscillators regulate circadian gene expression in Neurospora. Proc. Natl. Acad. Sci. 100: $13597-13602$.

Crosthwaite, S.K., Loros, J.J., and Dunlap, J.C. 1995. Light-induced resetting of a circadian clock is mediated by a rapid increase in frequency transcript. Cell 81: 1003-1012.

Crosthwaite, S.K., Dunlap, J.C., and Loros, J.J. 1997. Neurospora $w c-1$ and $w c-2$ : Transcription, photoresponses, and the origins of circadian rhythmicity. Science 276: 763-769.

Davis, M., Hatzubai, A., Andersen, J.S., Ben-Shushan, E., Fisher, G.Z., Yaron, A., Bauskin, A., Mercurio, F., Mann, M., and Ben-Neriah, Y. 2002. Pseudosubstrate regulation of the $\mathrm{SCF}(\beta-\mathrm{TrCP})$ ubiquitin ligase by hnRNP-U. Genes \& Dev. 16: 439-451.

De Leersnyder, H., De Blois, M.C., Claustrat, B., Romana, S., Albrecht, U., Von Kleist-Retzow, J.C., Delobel, B., Viot, G., Lyonnet, S., Vekemans, M., et al. 2001. Inversion of the circadian rhythm of melatonin in the Smith-Magenis syndrome. J. Pediatr. 139: 111-116.

Denault, D.L., Loros, J.J., and Dunlap, J.C. 2001. WC-2 mediates WC-1-FRQ interaction within the PAS protein-linked circa- dian feedback loop of Neurospora. EMBO J. 20: 109-117.

Deng, X.W., Dubiel, W., Wei, N., Hofmann, K., Mundt, K., Colicelli, J., Kato, J., Naumann, M., Segal, D., Seeger, M., et al. 2000. Unified nomenclature for the COP9 signalosome and its subunits: An essential regulator of development. Trends Genet. 16: 202-203.

Dunlap, J.C. 1999. Molecular bases for circadian clocks. Cell 96: $271-290$.

Dunlap, J.C. and Loros, J.J. 2004. The Neurospora circadian system. J. Biol. Rhythms 19: 414-424.

Edery, I., Zweibel, L., Dembinska, M., and Rosbash, M. 1994. Temporal phosphorylation of the Drosophila period protein. Proc. Nat1. Acad. Sci. 91: 2260-2264.

Eide, E.J., Woolf, M.F., Kang, H., Woolf, P., Hurst, W., Camacho, F., Vielhaber, E.L., Giovanni, A., and Virshup, D.M. 2005. Control of mammalian circadian rhythm by CKI $\varepsilon$-regulated proteasome-mediated PER2 degradation. Mol. Cell. Biol. 25: 2795-2807.

Elsea, S.H., Mykytyn, K., Ferrell, K., Coulter, K.L., Das, P., Dubiel, W., Patel, P.I., and Metherall, J.E. 1999. Hemizygosity for the COP9 signalosome subunit gene, SGN3, in the Smith-Magenis syndrome. Am. J. Med. Genet. 87: 342-348.

Feng, S., Shen, Y., Sullivan, J.A., Rubio, V., Xiong, Y., Sun, T.P., and Deng, X.W. 2004. Arabidopsis CAND1, an unmodified CUL1-interacting protein, is involved in multiple developmental pathways controlled by ubiquitin/proteasome-mediated protein degradation. Plant Cell 16: 1870-1882.

Froehlich, A.C., Loros, J.J., and Dunlap, J.C. 2003. Rhythmic binding of a WHITE COLLAR-containing complex to the frequency promoter is inhibited by FREQUENCY. Proc. Natl. Acad. Sci. 100: 5914-5919.

Garceau, N., Liu, Y., Loros, J.J., and Dunlap, J.C. 1997. Alternative initiation of translation and time-specific phosphorylation yield multiple forms of the essential clock protein FREQUENCY. Cell 89: 469-476.

Goldenberg, S.J., Cascio, T.C., Shumway, S.D., Garbutt, K.C., Liu, J., Xiong, Y., and Zheng, N. 2004. Structure of the Cand1-Cul1-Roc1 complex reveals regulatory mechanisms for the assembly of the multisubunit cullin-dependent ubiquitin ligases. Cell 119: 517-528.

Gorl, M., Merrow, M., Huttner, B., Johnson, J., Roenneberg, T., and Brunner, M. 2001. A PEST-like element in FREQUENCY determines the length of the circadian period in Neurospora crassa. EMBO I. 20: 7074-7084.

Granshaw, T., Tsukamoto, M., and Brody, S. 2003. Circadian rhythms in Neurospora crassa: Farnesol or geraniol allow expression of rhythmicity in the otherwise arrhythmic strains frq10, wc-1, and wc-2. J. Biol. Rhythms 18: 287-296.

Greenberg, F., Lewis, R.A., Potocki, L., Glaze, D., Parke, J., Killian, J., Murphy, M.A., Williamson, D., Brown, F., Dutton, R., et al. 1996. Multi-disciplinary clinical study of SmithMagenis syndrome (deletion 17p11.2). Am. J. Med. Genet. 62: $247-254$.

Grima, B., Lamouroux, A., Chelot, E., Papin, C., LimbourgBouchon, B., and Rouyer, F. 2002. The F-box protein slimb controls the levels of clock proteins period and timeless. Nature 420: 178-182.

He, Q., Cheng, P., Yang, Y., Wang, L., Gardner, K.H., and Liu, Y. 2002. White collar-1, a DNA binding transcription factor and a light sensor. Science 297: 840-843.

He, Q., Cheng, P., Yang, Y., He, Q., Yu, H., and Liu, Y. 2003. FWD1-mediated degradation of FREQUENCY in Neurospora establishes a conserved mechanism for circadian clock regulation. $E M B O ~ J .22: 4421-4430$.

Iwasaki, H. and Dunlap, J.C. 2000. Microbial circadian oscillatory systems in Neurospora and Synechococcus: Models for 
cellular clocks. Curr. Opin. Microbiol. 3: 189-196.

Ko, H.W., Jiang, J., and Edery, I. 2002. Role for Slimb in the degradation of Drosophila Period protein phosphorylated by Doubletime. Nature 420: 673-678.

Lakin-Thomas, P.L. and Brody, S. 2000. Circadian rhythms in Neurospora crassa: Lipid deficiencies restore robust rhythmicity to null frequency and white-collar mutants. Proc. Nat1. Acad. Sci. 97: 256-261.

Lee, C., Etchegaray, J.P., Cagampang, F.R., Loudon, A.S., and Reppert, S.M. 2001. Posttranslational mechanisms regulate the mammalian circadian clock. Cell 107: 855-867.

Li, L. and Deng, X.W. 2003. The COP9 signalosome: An alternative lid for the 26S proteasome? Trends Cell Biol. 13: 507509.

Lin, J.M., Kilman, V.L., Keegan, K., Paddock, B., Emery-Le, M., Rosbash, M., and Allada, R. 2002. A role for casein kinase $2 \alpha$ in the Drosophila circadian clock. Nature 420: 816-820.

Liu, Y. 2005. Analysis of posttranslational regulations in the neurospora circadian clock. Methods Enzymol. 393: 379393.

Liu, Y., Garceau, N., Loros, J.J., and Dunlap, J.C. 1997. Thermally regulated translational control mediates an aspect of temperature compensation in the Neurospora circadian clock. Cell 89: 477-486.

Liu, Y., Merrow, M.M., Loros, J.J., and Dunlap, J.C. 1998. How temperature changes reset a circadian oscillator. Science 281: 825-829.

Liu, Y., Loros, J., and Dunlap, J.C. 2000. Phosphorylation of the Neurospora clock protein FREQUENCY determines its degradation rate and strongly influences the period length of the circadian clock. Proc. Natl. Acad. Sci. 97: 234-239.

Liu, J., Furukawa, M., Matsumoto, T., and Xiong, Y. 2002. NEDD8 modification of CUL1 dissociates p120(CAND1), an inhibitor of CUL1-SKP1 binding and SCF ligases. Mol. Cell 10: $1511-1518$.

Liu, C., Powell, K.A., Mundt, K., Wu, L., Carr, A.M., and Caspari, T. 2003. Cop9/signalosome subunits and Pcu4 regulate ribonucleotide reductase by both checkpoint-dependent and -independent mechanisms. Genes \& Dev. 17: 11301140.

Loros, J.J. and Feldman, J.F. 1986. Loss of temperature compensation of circadian period length in the frq-9 mutant of Neurospora crassa. J. Biol. Rhythms 1: 187-198.

Lowrey, P.L., Shimomura, K., Antoch, M.P., Yamazaki, S., Zemenides, P.D., Ralph, M.R., Menaker, M., and Takahashi, J.S. 2000. Positional syntenic cloning and functional characterization of the mammalian circadian mutation tau. Science 288: 483-492.

Lyapina, S., Cope, G., Shevchenko, A., Serino, G., Tsuge, T., Zhou, C., Wolf, D.A., Wei, N., Shevchenko, A., and Deshaies, R.J. 2001. Promotion of NEDD-CUL1 conjugate cleavage by COP9 signalosome. Science 292: 1382-1385.

Merrow, M., Brunner, M., and Roenneberg, T. 1999. Assignment of circadian function for the Neurospora clock gene frequency. Nature 399: 584-586.

Merrow, M., Franchi, L., Dragovic, Z., Gorl, M., Johnson, J., Brunner, M., Macino, G., and Roenneberg, T. 2001. Circadian regulation of the light input pathway in Neurospora crassa. EMBO J. 20: 307-315.

Mundt, K.E., Porte, J., Murray, J.M., Brikos, C., Christensen, P.U., Caspari, T., Hagan, I.M., Millar, J.B., Simanis, V., Hofmann, K., et al. 1999. The COP9/signalosome complex is conserved in fission yeast and has a role in $\mathrm{S}$ phase. Curr. Biol. 9: 1427-1430.

Mundt, K.E., Liu, C., and Carr, A.M. 2002. Deletion mutants in COP9/signalosome subunits in fission yeast Schizosaccha- romyces pombe display distinct phenotypes. Mol. Biol. Cell 13: 493-502.

Petroski, M.D. and Deshaies, R.J. 2005. Function and regulation of cullin-RING ubiquitin ligases. Nat. Rev. Mol. Cell Biol. 6: 9-20.

Potocki, L., Chen, K.S., and Lupski, J.R. 1999. Subunit 3 of the COP9 signal transduction complex is conserved from plants to humans and maps within the Smith-Magenis syndrome critical region in 17p11.2. Genomics 57: 180-182.

Potocki, L., Glaze, D., Tan, D.X., Park, S.S., Kashork, C.D., Shaffer, L.G., Reiter, R.J., and Lupski, J.R. 2000. Circadian rhythm abnormalities of melatonin in Smith-Magenis syndrome. J. Med. Genet. 37: 428-433.

Pregueiro, A.M., Price-Lloyd, N., Bell-Pedersen, D., Heintzen, C., Loros, J.J., and Dunlap, J.C. 2005. Assignment of an essential role for the Neurospora frequency gene in circadian entrainment to temperature cycles. Proc. Natl. Acd. Sci. 102: 2210-2215.

Sathyanarayanan, S., Zheng, X., Xiao, R., and Sehgal, A. 2004. Posttranslational regulation of Drosophila PERIOD protein by protein phosphatase 2A. Cell 116: 603-615.

Schwechheimer, C. 2004. The COP9 signalosome (CSN): An evolutionary conserved proteolysis regulator in eukaryotic development. Biochim. Biophys. Acta 1695: 45-54.

Schwechheimer, C., Serino, G., Callis, J., Crosby, W.L., Lyapina, S., Deshaies, R.J., Gray, W.M., Estelle, M., and Deng, X.W. 2001. Interactions of the COP9 signalosome with the E3 ubiquitin ligase SCFTIRI in mediating auxin response. Science 292: 1379-1382.

Sizemore, S.T. and Paietta, J.V. 2002. Cloning and characterization of scon-3+, a new member of the Neurospora crassa sulfur regulatory system. Eukaryot. Cell 1: 875-883.

Smith, A.C., Dykens, E., and Greenberg, F. 1998. Sleep disturbance in Smith-Magenis syndrome (del 17 p11.2). Am. J. Med. Genet. 81: 186-191.

Talora, C., Franchi, L., Linden, H., Ballario, P., and Macino, G. 1999. Role of a white collar-1-white collar-2 complex in blue-light signal transduction. EMBO J. 18: 4961-4968.

Wee, S., Geyer, R.K., Toda, T., and Wolf, D.A. 2005. CSN facilitates Cullin-RING ubiquitin ligase function by counteracting autocatalytic adapter instability. Nat. Cell Biol. 7: 387-391.

Wei, N. and Deng, X.W. 2003. The COP9 signalosome. Annu. Rev. Cell Dev. Biol. 19: 261-286.

Wei, N., Chamovitz, D.A., and Deng, X.W. 1994. Arabidopsis COP9 is a component of a novel signaling complex mediating light control of development. Cell 78: 117-124.

Wolf, D.A., Zhou, C., and Wee, S. 2003. The COP9 signalosome: An assembly and maintenance platform for cullin ubiquitin ligases? Nat. Cell Biol. 5: 1029-1033.

Yang, Y., Cheng, P., Zhi, G., and Liu, Y. 2001. Identification of a calcium/calmodulin-dependent protein kinase that phosphorylates the Neurospora circadian clock protein FREQUENCY. J. Biol. Chem. 276: 41064-41072.

Yang, X., Menon, S., Lykke-Andersen, K., Tsuge, T., Di, X., Wang, X., Rodriguez-Suarez, R.J., Zhang, H., and Wei, N. 2002. The COP9 signalosome inhibits p27(kip1) degradation and impedes G1-S phase progression via deneddylation of SCF Cul1. Curr. Biol. 12: 667-672.

Yang, Y., Cheng, P., and Liu, Y. 2002. Regulation of the Neurospora circadian clock by casein kinase II. Genes \& Dev. 16: 994-1006.

Yang, Y., Cheng, P., He, Q., Wang, L., and Liu, Y. 2003. Phosphorylation of FREQUENCY protein by casein kinase II is necessary for the function of the Neurospora circadian clock. Mol. Cell. Biol. 23: 6221-6228. 
Yang, Y., He, Q., Cheng, P., Wrage, P., Yarden, O., and Liu, Y. 2004. Distinct roles for PP1 and PP2A in the Neurospora circadian clock. Genes \& Dev. 18: 255-260.

Young, M.W. and Kay, S.A. 2001. Time zones: A comparative genetics of circadian clocks. Nat. Rev. Genet. 2: 702715.

Zheng, J., Yang, X., Harrell, J.M., Ryzhikov, S., Shim, E.H., Lykke-Andersen, K., Wei, N., Sun, H., Kobayashi, R., and Zhang, H. 2002. CAND1 binds to unneddylated CUL1 and regulates the formation of SCF ubiquitin E3 ligase complex. Mol. Cell 10: 1519-1526.

Zhou, P. and Howley, P.M. 1998. Ubiquitination and degradation of the substrate recognition subunits of SCF ubiquitinprotein ligases. Mol. Cell 2: 571-580.

Zhou, C., Wee, S., Rhee, E., Naumann, M., Dubiel, W., and Wolf, D.A. 2003. Fission yeast COP9/signalosome suppresses cullin activity through recruitment of the deubiquitylating enzyme Ubp12p. Mol. Cell 11: 927-938. 


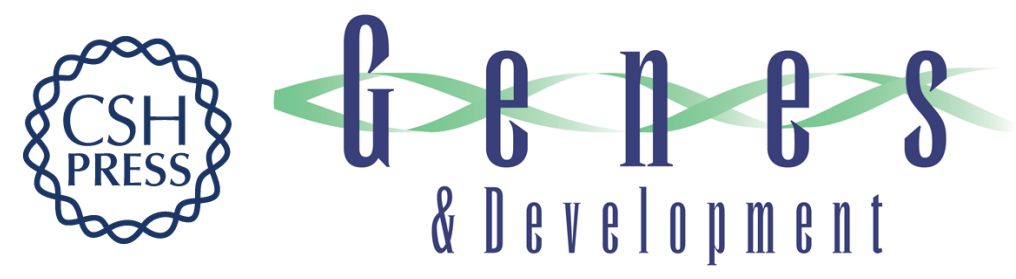

\section{The COP9 signalosome regulates the Neurospora circadian clock by controlling the stability of the SCF FWD-1 complex}

Qun He, Ping Cheng, Qiyang He, et al.

Genes Dev. 2005, 19:

Access the most recent version at doi:10.1101/gad.1322205

References This article cites 74 articles, 35 of which can be accessed free at: http://genesdev.cshlp.org/content/19/13/1518.full.html\#ref-list-1

License

Email Alerting

Receive free email alerts when new articles cite this article - sign up in the box at the top Service right corner of the article or click here.

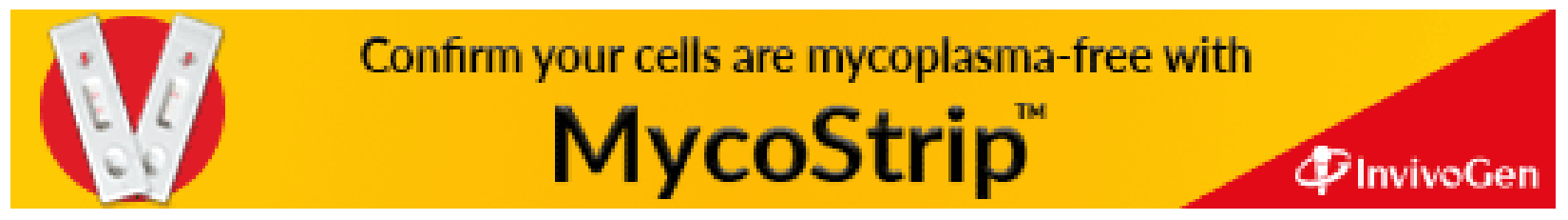

